\title{
Preparo de Cólon para Colonoscopia com Polietilenoglicol versus Sulfato de Magnésio em Pacientes acima de 70 anos de Idade
}

\author{
Bowel Preparation for Colonoscopy: A Comparison of Polyethylene Glycol and \\ Magnesium Sulphate in Patients Over 70 Years Old
}

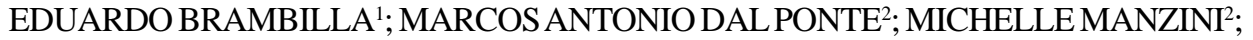 \\ ROBERTOTABOADAFELLINI²; VIVIANE RAQUEL BUFFON²; RAFAEL SCHALLINS MAY ${ }^{2}$ \\ ${ }^{1}$ Professor da UEM Aparelho Digestivo da Universidade de Caxias do Sul; Membro Titular da Sociedade \\ Brasileira de Coloproctologia; ${ }^{2}$ Acadêmicos do curso de Medicina da Universidade de Caxias do Sul.
}

\begin{abstract}
BRAMBILLA E; DAL PONTE MA; MANZINI M; FELLINI RT; BUFFON VR; MAY RS. Preparo de Cólon para Colonoscopia com Polietilenoglicol versus Sulfato de Magnésio em Pacientes acima de 70 anos de Idade. Rev bras Coloproct, 2008;28(2): $204-209$.

RESUMO: Objetivos: Comparar a eficácia e segurança do sulfato de magnésio em relação ao polietilenoglicol no preparo intestinal para colonoscopia em pacientes com idade igual ou superior a 70 anos. Métodos: Foram selecionados para o estudo sessenta pacientes acima de $\mathbf{7 0}$ anos, de ambos os sexos e que foram aleatoriamente divididos em dois grupos: o grupo controle, utilizando o polietilenoglicol e o grupo em estudo com sulfato de magnésio. Foram avaliados a qualidade do preparo, a tolerância pelos pacientes e os efeitos adversos produzidos por cada substância. $O$ colonoscopista possuía informação prévia sobre o tipo de preparo. Resultados: Os pacientes apresentaram uma distribuição homogênea nos grupos e nos fatores idade, patologias prévias, efeitos colaterais, qualidade de preparo e tempo de chegada ao ceco na realização da colonoscopia. Diferiram na distribuição do sexo, apresentando um maior número de indivíduos do sexo feminino no grupo que utilizou o sulfato de magnésio, porém sem relevância estatística. Conclusão: $O$ sulfato de magnésio não demonstrou diferença estatística quanto aos níveis de segurança e eficácia em pacientes maiores de 70 anos, para o preparo intestinal, quando comparado ao polietilenoglicol.
\end{abstract}

Descritores: Cólon, Colonoscopia, Diagnóstico, Neoplasias do Colo, Sulfato de Magnésio.

\section{INTRODUÇÃO}

A colonoscopia é o melhor exame de imagem para o diagnóstico e para a triagem do câncer colorretal, assim como, para a avaliação de várias patologias do cólon. É de grande importância a qualidade do preparo intestinal, sendo isto, essencial para a visualização adequada de toda a superfície colônica evitando que pequenas lesões sejam negligenciadas ${ }^{1}$. Além disso o preparo intestinal adequado diminui o tempo de duração da colonoscopia, bem como seus custos ${ }^{2}$.
Os idosos são os pacientes mais propensos ao câncer colorretal e a outras patologias do cólon. Esta faixa etária pode apresentar menor tolerabilidade ao preparo colônico, sobretudo caso tiverem comorbidades associadas. O resultado destas condições leva ao aumento dos efeitos adversos causados pelo preparo, bem como, acarretam a limpeza inadequada do intestino grosso, dificultando o exame ${ }^{3}$.

O método de preparo ideal deve apresentar vantagens em relação à eficácia, à segurança, ao custo, à facilidade de administração e à tolerância do paci-

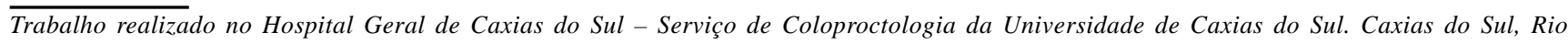
Grande do Sul, Brasil.

Conflito de interesses: As medicações usadas na pesquisa foram doadas pelo laboratório Libbs, na forma de medicação a ser disponibilizada aos pacientes.

Este estudo foi aprovado pela Comissão de Ética do Hospital Geral de Caxias do Sul. 
ente, contudo ainda não está disponível uma substância que apresente todas estas qualidades ${ }^{4,5}$.

Os preparos mais amplamente utilizados na literatura mundial são o polietilenoglicol e o fosfato de sódio, o qual, apesar de sua eficácia, acarreta desequilíbrio hidroeletrolítico devendo ser evitado em idosos predispostos a estes distúrbios, além de apresentar sabor ruim, o que não necessariamente reduz a aceitação pelo paciente ${ }^{2,6}$.

A solução oral de polietilenoglicol é um dos preparos colônicos mais utilizados. Trata-se de uma solução inabsorvível e iso-osmolar com o plasma, sendo segura, na medida em que não há absorção ou excreção de água e de eletrólitos ${ }^{7}$. Apresenta boa eficácia e tolerância ${ }^{3}$, contudo os pacientes queixam-se do grande volume a ser ingerido ${ }^{8}$. Entre os efeitos adversos, mencionam-se dor abdominal e náuseas. $\mathrm{O}$ regime de duas doses, com uma na noite anterior e outra na manhã que precede o exame melhora sua aceitação e a aderência por parte do paciente, além de mostrar-se superior quanto à lavagem do cólon, quando comparado com regimes de dose única ${ }^{9,10}$. Foi demonstrado também, que o regime de duas doses, sem restrição na dieta, melhora a qualidade da lavagem do cólon, sem interferir na tolerabilidade do preparo pelo paciente ${ }^{11}$.

O sulfato de magnésio é um laxativo salino que atua extraindo líquido da luz intestinal, ao criar um gradiente osmótico através da parede intestinal ${ }^{12,13}$. Esta substância é muito utilizada em nosso meio de forma leiga como laxativo, e pode ser dispensada sem receita médica a um custo extremamente baixo.

O objetivo deste estudo é comparar qual é a melhor substância para emprego no preparo de cólon: polietilenoglicol ou sulfato de magnésio, avaliando-se o preparo obtido, os efeitos colaterais, a facilidade de administração dos agentes e o custo em pacientes idosos.

\section{PACIENTES E MÉTODOS}

Os pacientes incluídos neste estudo foram escolhidos entre os indivíduos que procuraram o Serviço de Colonoscopia do Hospital Geral de Caxias do Sul, em regime ambulatorial, de ambos os sexos e com idade igual ou superior a 70 anos no período de 18 de setembro até 30 de dezembro de 2006. Os critérios de exclusão foram: pacientes internados por qualquer patologia, portadores de insuficiência renal crônica gra- ve, insuficiência cardíaca descompensada, ascite, hemorragia digestiva, evento vascular isquêmico recente e cirurgia colônica prévia.

De acordo com a seqüência de agendamento do procedimento, os pacientes que preencheram os critérios acima e que aceitaram participar do estudo, mediante assinatura de consentimento informado, foram selecionados. Participaram do estudo 60 pacientes, de ambos os sexos e com idade média de 75 anos.

Aleatoriamente, os pacientes foram alocados para um dos dois grupos: grupo controle - preparo intestinal com polietilenoglicol - e grupo em estudo - com sulfato de magnésio - , sem estarem cientes de qual preparo estavam usando.

Ambos os grupos receberam dieta líquida no dia anterior ao exame. O grupo que usou polietilenoglicol ingeriu, no dia anterior ao exame, 1 litro de polietilenoglicol às $16,18,20$ e 22 horas. Os indivíduos que usaram sulfato de magnésio ingeriram $15 \mathrm{~g}$ da substância misturada com suco de laranja às 10, 14, 18 e 22 horas do dia anterior ao procedimento. No dia do procedimento, os pacientes em ambos os preparos permaneceram em NPO até a realização do exame. O exame foi realizado sob sedação com Midazolam $3 \mathrm{mg}$ associado à Petidina $30 \mathrm{mg}$.

O examinador era coloproctologista com experiência mínima de 3000 exames, e não sabia previamente qual dos dois preparos havia sido utilizado pelo paciente.

O paciente respondeu um questionário após o uso do preparo, e imediatamente antes da realização do exame. Foi avaliada a tolerância quanto ao preparo e a necessidade de interrompê-lo. Sintomas específicos como dor abdominal, náuseas, vômitos, tonturas, diarréia, fraqueza e desmaio foram também questionados. A intensidade da cólica foi graduada de 1 a 10, em ordem crescente de intensidade por escala de dor. O sabor do preparo foi graduado em bom, regular e ruim. Os pacientes que já haviam realizado colonoscopia prévia responderam sobre a preferência do preparo usado neste estudo ou naquele utilizado anteriormente, quando os preparos não eram os mesmos.

O examinador preencheu um formulário para cada paciente. A qualidade do preparo foi denominada como boa, regular e ruim. Quantificou-se o volume aspirado. Mediu-se o tempo de duração da colonoscopia, sendo este cronometrado, a partir da introdução do colonoscópio no canal anal até a chegada deste ao ceco. A dificuldade do exame foi graduada 
em fácil, moderada e difícil. Por último, relatou os achados do exame.

\section{ANÁLISE ESTATÍSTICA}

Os dados são apresentados como valores médios e desvios-estândares. Foi usado o método estatístico do qui-quadrado para comparar escalas nominais, com o teste exato de Fischer quando indicado. O teste de Hosmer e Lemeshow foi utilizado para verificar a homogeneidade da amostra.

Para todos os testes, o valor de $5 \%(\mathrm{p}<0,05)$ ou inferior foi considerado como sendo significativo.

Todos os dados foram tabulados pelo software SPSS versão 11.5 para Windows (Microsoft Corp, Redmond, Wash).

\section{RESULTADOS}

Os 60 pacientes selecionados para o estudo foram aleatoriamente alocados para um dos dois grupos: $39(65 \%)$ pacientes para o sulfato de magnésio (estudo) e 21(35\%) para o polietilenoglicol (controle). O grupo em estudo era composto por 31 mulheres e por 8 homens, já entre os pacientes do grupo controle, havia 12 mulheres e 9 homens. As idades dos pacientes variaram entre 70 e 86 anos $(75,48 \pm 4,60)$. As comorbidades avaliadas estavam distribuídas de maneira similar em ambos os grupos, bem como a realização de cirurgias prévias. Tabela 1 .

No grupo do sal amargo, 7 (33\%) pacientes e $3(8 \%)$ dos indivíduos do grupo do polietilenoglicol não conseguiram concluir o preparo devido a efeitos colaterais, principalmente náuseas e vômitos. Cólicas abdominais apareceram em 4 pacientes $(10 \%)$ e desidratação leve a moderada em $3(8 \%)$ daqueles pertencentes ao grupo que utilizou o sulfato de magnésio. Houve 1 caso de desmaio no grupo controle, porém sem demonstrar diferença significativa. Tabela 2.

$\mathrm{Na}$ avaliação do colonoscopista o preparo foi considerado bom a regular em $85 \%$ dos casos e ruim em $15 \%$ dos casos. Obteve-se intubação do ceco em todos os exames com tempo médio de 8,5 minutos, variando de 3 até 40 minutos. O grau de dificuldade apontado pelo colonoscopista foi de fácil a moderada em $92 \%$ dos casos e difícil $8 \%$. Não houve diferença entre os grupos quanto à avaliação do grau de dificuldade, tempo de chegada ao ceco, qualidade do preparo e no volume de líquido aspirado. Figura 1.

Do total de pacientes, 34 (57\%) consideraram o sabor do preparo bom; 20 (33\%), regular; e 6 (10\%) pacientes como sendo ruim. Tais valores não demonstraram diferença estatisticamente significativa entre ambos os grupos. Figura 2.

Nesta amostra, $39(65 \%)$ pacientes estavam fazendo a colonoscopia pela primeira vez. Os 20 (35\%) pacientes restantes foram solicitados a comparar o preparo atual com aquele usado previamente. $\mathrm{O}$ sal amargo foi o preferido por $10(50 \%)$, seguido pelo manitol, com $3(15 \%)$. O polietilenoglicol foi apontado como o favorito por $2(10 \%)$ indivíduos e o picossulfato de sódio, por apenas $1(5 \%)$ e $4(20 \%)$ pacientes que já haviam realizado o exame anteriormente, não notaram diferença entre os preparos.

Tabela 1 - Características dos pacientes. P: NS

\begin{tabular}{lccccc}
\hline Preparo & $\begin{array}{c}\text { Média } \\
\text { de Idade }\end{array}$ & $\begin{array}{c}\text { Sexo } \\
\text { Masculino }\end{array}$ & $\begin{array}{c}\text { Sexo } \\
\text { Feminino }\end{array}$ & Co-morbidades & $\begin{array}{c}\text { Cirurgias } \\
\text { Prévias }\end{array}$ \\
\hline Polietilenoglicol & 75 anos & $43 \%$ & $57 \%$ & $15 \%$ & $21 \%$ \\
Sulfato de magnésio & 75 anos & $21 \%$ & $79 \%$ & $28 \%$ & $39 \%$ \\
\hline
\end{tabular}

Tabela 2 - Efeitos adversos distribuídos nos grupos estudados. P: NS

\begin{tabular}{lccccccc}
\hline Efeitos & Cólicas & Náuseas & Vômitos & Fraqueza & Interrupção & Desidratação & Desmaio \\
\hline Sulfato de Magnésio & $10 \%$ & $33 \%$ & $13 \%$ & $33 \%$ & $8 \%$ & $8 \%$ & - \\
Polietilenoglicol & - & $43 \%$ & $10 \%$ & $19 \%$ & $33 \%$ & - & $4 \%$ \\
\hline
\end{tabular}




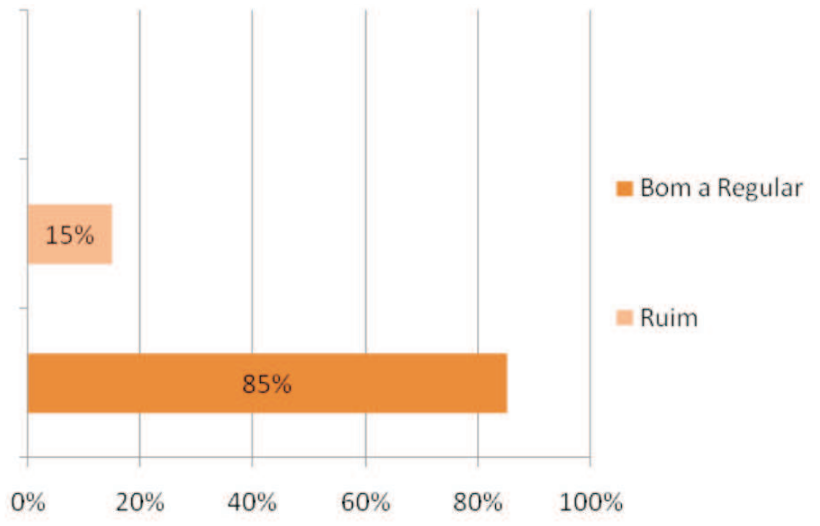

Figura 1 - Qualidade do preparo segundo colonoscopista.

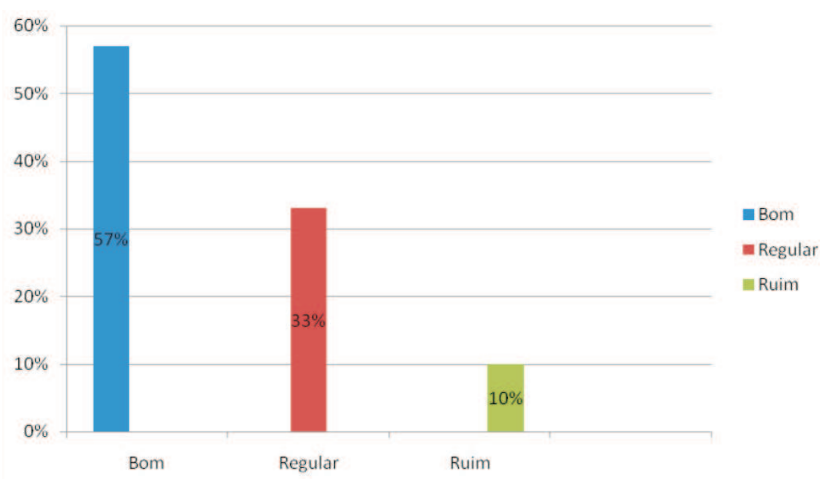

Figura 2 - Resultados quanto ao sabor do preparo. P: NS

\section{DISCUSSÃO}

Uma vez que a colonoscopia é o principal exame diagnóstico para doenças do cólon, é de suma importância que o preparo intestinal seja adequado. $\mathrm{O}$ volume de 4 litros de polietilenoglicol mostrou ser tão eficaz quanto $750 \mathrm{~mL}$ de sulfato de magnésio, em relação à qualidade do preparo colônico. Nenhuma das suas substâncias ocasionou maior freqüência, nem maior gravidade de efeitos adversos.

Um fator de relevância a ser considerado é o valor de cada preparo. O polietilenoglicol custa, em média, $\mathrm{R} \$ 36$ para que sejam ingeridos os 4 litros necessários para a realização do exame, já para os $60 \mathrm{~g}$ de sulfato de magnésio a serem diluídos em $750 \mathrm{~mL}$ de líquido, tem um custo de cerca de $\mathrm{R} \$ 4,50$.

Apesar de haver relato de caso, no qual agentes hiperosmóticos, como citrato de magnésio e fosfato de sódio, estão relacionados à colite isquêmica ${ }^{13}$, não foi observado esse efeito adverso em nosso estudo.
Os pacientes do grupo do sulfato de magnésio não apresentaram sintomatologia ocasionada por distúrbios hidroeletrolíticos, contudo os pacientes com insuficiência renal grave, ascite e cardiopatia isquêmica foram excluídos do estudo por não sabermos a real segurança dessa substância nesses grupos de pacientes.

Não houve complicações significativas durante o preparo nem durante a colonoscopia. Há estudos em pacientes idosos ${ }^{3}$, relatando a ausência de tais complicações ao se utilizar o polietilenoglicol e o sulfato de sódio como substâncias para o preparo. Com o presente estudo, pode-se afirmar a mesma segurança com o sulfato de magnésio.

Entre os pacientes que já haviam sido submetidos ao exame colonoscópico previamente, a maioria preferiu o sulfato de magnésio, como a substância para preparo com o melhor sabor. A tolerância quanto ao volume a ser ingerido foi semelhante, quando comparados ambos os grupos.

Do total de pacientes, um paciente $(2 \%)$ apenas apresentou desmaio, havendo ingerido polietilenoglicol para o preparo intestinal, entretanto esse advento não foi relacionado à desidratação clinicamente evidente, e tampouco tem relevância estatística.

Apesar de haverem dados na literatura, afirmando que as cólicas abdominais são efeitos adversos provocados pelo polietilenoglicol, nenhum paciente desse grupo as apresentou, além disso a diferença entre os dois grupos não foi significante.

Embora houvesse muito mais pacientes do sexo feminino participando do estudo, os ajustes realizados mediante métodos estatísticos comprovaram a ausência de influência estatisticamente significativa sobre os resultados.

\section{CONCLUSÕES}

O estudo não demonstrou diferença em relação à eficácia do preparo intestinal, tanto para o polietilenoglicol, como para o sulfato de magnésio. Não houve predomínio de frequiência nem de severidade de efeitos adversos por parte de qualquer uma das soluções. Ambos os preparos mostraram-se seguros para pacientes com idade superior a 70 anos.

Recomenda-se o uso do sulfato de magnésio para o preparo colonoscópico, principalmente, em nível de saúde pública, quando é necessária a contenção 
de gastos, uma vez que seu custo é muito inferior ao do polietilenoglicol. Outra vantagem do sal amargo é o menor volume ingerido por paciente, porém mais estudos, com grupos maiores de pacientes, devem ser realizados para avaliação de efeitos colaterais menos freqüentes.

\section{AGRADECIMENTOS}

Agradecemos ao Prof. Dr. Wilson Spiandorello, professor da Universidade de Caxias do Sul por sua valiosa colaboração na avaliação dos dados estatísticos.

ABSTRACT: Objectives: To compare efficacy and safety of magnesium sulphate in relation to polyethylene glycol in bowel preparation for colonoscopy. Methods: Sixty patients older than 70 years old, of both genders, were selected for the study, and were randomly divided into two groups: the control group, using polyethylene glycol and the in study group with magnesium sulphate. Quality of preparation, patient tolerance, and side effects produced by each substance were evaluated. Colonoscopist was blinded in relation to the information of bowel preparation. Results: Patients present a homogeneus distribution in groups, in factors as age, past pathologies, side effects, cleansing quality and time taken to reach cecum during colonoscopy. There were differences in gender distribution, being higher the number of female individuals in the group which used magnesium sulphate, however with no statistical significance. Conclusion: Magnesium sulphate presented no statistical difference in relation to safety and efficacy levels in patients older than $\mathbf{7 0}$ years old, for bowel preparation, when it is compared with polyethylene glycol.

Key words: Colon, Colonoscopy, Diagnosis, Colon Neoplasms, Magnesium Sulphate.

\section{REFERÊNCIAS}

1. Harewood G C, Sharma V K, Garmo P - Impact of colonoscopy preparation quality on detection of suspected colonic neoplasia. Gastrointest Endosc 2003; 53(1): p 76-79.

2. Kositchaiwat S, Suwanthanmma W, Suvikapakornkul R, Tiewtahnom V, Rerkpatanakit P, Tinkornrusmee C Comparative study of two bowel preparaton regimens for colonoscopy: Senna tablets vs sodium phosphate solution. World J Gastroenterol 2006; 12(34): p 55365539.

3. Seinelä L, Pehknone E, Laasanen T, Ahvenainen J Bowel preparation for colonoscopy in very old patients: a randomized prospective trial comparing oral sodium phosphate and polyethylene glycol electrolyte lavage solution. Scand J Gastroenterol 2003; 38(2): p 216220.

4. Parra-Blanco A, Nicolás-Pérez D, Gimeno-García A, Grosso B, Jiménez A, Ortega $J$ et al - The timing of bowel preparation before colonoscopy determines the quality of cleansing, and is a significant factor contributing to the detection of flat lesions: A randomized study. World J Gastroenterol 2006; 12(38): p 6161-6166.

5. Habr-Gama A, Bringel R W A, Nahas S G, Araújo S E A, Souza Junior A H, Calache J E et al - Bowel preparation for colonoscopy: a comparison of manitol and sodium phosphate: Results of a prospective randomized study.
Rev Hops Clin Fac Med S Paulo 1999; 54(6): p 187192.

6. Miki Jr P, Rocha J J R, Aprilli F, Féres O - Estudo comparativo entre as soluções de manitol, picossulfato de sódio e fosfato monobásico e dibásico de sódio no preparo de cólon para a colonoscopia. Acta Cir Brás 2002; 17(3): p 64-68.

7. Vligen-Mathus E M H, Kemble U M - A prospective randomized blinded comparison of sodium phosphate and polyethylene glycol-electrolyte solution for safe bowel cleansing. Aliment Pharmacol Ther 2006; 23: p 543-552.

8. Law W-L, Choi H-K, Chu K-W, Ho J W C, Wong L - Bowel Preparation for Colonoscopy: A Randomized Controlled Trial Comparing Polyethylene Glycol Solution, One Dose and Two Doses of Oral Sodium Phosphate Solution. Asian J Surg 2004; 27(2): p 120-124.

9. Ell C, Fischbach W, Keller R, Dehe M, Mayer G, Schneider B et al - A randomized, blinded, prospective trial to compare the safety and efficacy of three bowel-cleansing solutions for colonoscopy. Endosc 2003; 35(4): p 300-304.

10. Huppertz-Haus G, Bretthauer M, Sauar J, Paulsen J, Kjellevold O, Majak B et al - Polyethylene glycol and sodium phosphate for bowel cleansing for colonoscopy: a randomized trial. Endosc 2005; 37(6): p 537-541.

11. Aoun E, Abdul-Baki H, Azar C, Mourad F, Barada K, Berro Z et al - A randomized single-blind trial of split-dose PEGelectrolyte solution without dietary restriction compared with whole dose PEG-electrolyte solution with dietary restriction for Colonoscopy Preparation. Gastrointest Endosc 2005; 62: p 213-218. 
Eduardo Brambilla e Cols.

12. Robert FS - Mechanism of Purgative Effect of Magnesium Sulphate on Mouse Colon. J Diarrhoeal Dis Res 1997; 15(4): p 248-249.

13. Oh J K, Meiselman M, Lataif L E - Ischemic colitis caused by hyperosmotic saline laxatives. Gastrointet endosc 1997; 45(3): p 319-322.
Endereço para correspondência:

EDUARDO BRAMBILLA

Rua General Arcy da Rocha Nóbrega, 401/705 - Madureira

Caxias do Sul-RS

CEP: 95040-000

Fone/Fax: (54) 32229874

E-mail: brambilla.procto@terra.com.br 